Int. J. Odontostomat., 4(1):65-70, 2010.

\title{
Maloclusiones y su Impacto Psicosocial en Estudiantes de un Liceo Intercultural
}

\author{
Malocclusions and its Psychosocial Impact in Intercultural High-School Students
}

Ricardo Cartes-Velásquez*; Eric Araya** \& Carolina Valdés**

CARTES-VELÁSQUEZ, R.; ARAYA, E. \& VALDÉS, C. Maloclusiones y su impacto psicosocial en estudiantes de un liceo intercultural. Int. J. Odontostomat., 4(1):65-70, 2010.

RESUMEN: Las maloclusiones estan consideradas como la tercera patología oral de mayor prevalencia e impacto en el mundo, a pesar de esto son pocos los estudios en Chile que abordan el tema y consideran sus consecuencias en la calidad de vida o su relación con grupos poblacionales específicos. El objetivo de este estudio es determinar la prevalencia y su impacto psicosocial en jóvenes que estudian en un liceo con población indígena. Se realizó un estudio descriptivo de corte transversal, en 129 alumnos, 73,6\% pehuenche, del Liceo de Ralco; se utilizó el Índice Estetico Dental para cuantificar la magnitud de las maloclusiones y un Cuestionario de Autoimagen para evaluar el aspecto psicosocial. Los resultados muestran una prevalencia del $67,4 \%$ y un $21,7 \%$ para el nivel discapacitante de maloclusiones, siendo significativamente mayor en población pehuenche y rural, valores por sobre el de estadísticas nacionales e internacionales. A más de la mitad de los jóvenes las maloclusiones le ocasionan problemas para relacionarse con sus pares. Todo lo anterior hace necesario implementar estrategias preventivas y curativas que den solución a este problema.

PALABRAS CLAVE: maloclusión, estética dental, autoestima, psicosocial, indígena.

\section{INTRODUCCIÓN}

La prevalencia de las enfermedades bucales más frecuentes, caries y gingivitis, ha mostrado un mantenido descenso durante las últimas 2 décadas en nuestro país, esto al menos en los grupos de 6, 12 y menores de 20 años en general (Soto et al., 2007), donde se han priorizado los recursos y se han desarrollado estrategias tales como los Módulos Dentales de JUNAEB y el GES Odontológico de 6 años. Si bien dichas estrategias han logrado notables resultados, no han considerado a la tercera patología oral de mayor prevalencia e impacto en la población: las maloclusiones, que según Soto et al. en nuestro país afectarian al $52,5 \%$ de los niños de 12 años.

El efecto de las maloclusiones no es tan sólo funcional y/o estético, sino que también influye en los aspectos psicosociales (de Paula et al., 2009; Klages et al., 2006) y la calidad de vida, especialmente de los adolescentes (Taylor et al., 2009; Marques et al., 2006; Agou et al., 2008; Onyeaso \& Sanu, 2005). Diversas metodologías e índices (Hägg et al., 2007) se han desarrollado tanto para cuantificar la presencia y gravedad de las maloclusiones como para ver la relación e impacto sobre los aspectos estéticos y la calidad de vida; ante todas estas opciones la Organización Mundial de la Salud ha decidido optar por el Índice Estético Dental como instrumento evaluador (OMS, 1997), esto gracias a que combina tanto aspectos objetivos de necesidad de tratamiento ortodóncico como los estéticos subjetivos.

Si bien en países desarrollados las maloclusiones, y sus efectos ya mencionados, se encuentran ampliamente descritos en la literatura, en Chile este es un campo poco explorado. El objetivo de este trabajo es conocer la prevalencia y gravedad de las maloclusiones en adolescentes, relacionando el efecto psicosocial de estas, especialmente en las relaciones interpersonales con sus pares; e identificar las posibles diferencias entre la población de origen occidental e indígena, en este caso Pehuenche.

\footnotetext{
* Cirujano Dentista. Etapa de Destinación y Formación. CESFAM Ralco Alto Biobio. Servicio de Salud Biobio, Chile.

** Interno de Odontología. Universidad de Concepción, Chile.
} 


\section{MATERIAL Y MÉTODO}

Se realizó un estudio descriptivo de corte transversal en los estudiantes del Liceo Técnico Intercultural Bilingue de Ralco, Alto Biobio. Para ello se solicitaron las autorizaciones de la Dirección del Liceo y Departamento de Educación Municipal, así como el consentimiento de los jóvenes. De una matrícula de 144 alumnos, 129 accedieron a participar del estudio (Tabla I). Lo anterior significa que la muestra estudiada permitirá obtener una prevalencia con una precisión absoluta del $2,8 \%$, para un nivel de confianza del $95 \%$, utilizando una prevalencia del $52,5 \%$ basados en los datos entregados por Soto et al.

La recolección de datos se realizó en dependencias del Liceo, los escolares fueron sometidos a un examen clínico y respondieron un breve cuestionario, en un lugar habilitado especialmente para la ocasión, resguardando la tranquilidad necesaria. Un ejecutor y un asistente presenciaron solamente la toma de datos, los cuales fueron consignados en una ficha clínica elaborada para el presente trabajo, y así evitar sesgo por diferencias de criterio. Para la realización del examen clínico se utilizó sonda para caries, espe- jo número 5 y sonda periodontal, contando con luz natural adecuada para una correcta inspección visual, según los criterios de las OMS.

El Índice Estético Dental, IED, propuesto por la OPS/OMS fue escogido para la evaluación y clasificación de las anomalías dentofaciales.

Para la evaluación del impacto psicosocial se diseñó un Cuestionario de Autoimagen con 5 preguntas (Tabla II), enfocadas principalmente a conocer la influencia de las maloclusiones sobre la relación de los jóvenes con sus pares. Para la elaboración de este cuestionario se contó con la asesoría de los psicólogos y facilitadores interculturales del CESFAM Ralco, realizándose en forma previa al estudio una validación del instrumento con una muestra de 40 alumnos del mismo Liceo.

La tabulación de los datos se efectuó en el software MS Excel. Para el análisis estadístico se utilizo el test de $\mathrm{Chi}^{2}$ con un nivel de significación de $5 \%$ con el paquete estadístico Epidat 3.1.

Tabla I. Caracterización Etnodemográfica de los estudiantes del Liceo Intercultural de Ralco, Alto Biobio, Chile.

\begin{tabular}{lccccc}
\hline \multicolumn{5}{c}{ Sexo } & \multicolumn{3}{c}{ Etnia } \\
\hline Edad & Masculino & Femenino & Occidental & Pehuenche & Total \\
\hline 14 & 2 & 4 & 4 & 2 & 6 \\
15 & 10 & 13 & 4 & 19 & 23 \\
16 & 16 & 16 & 7 & 25 & 32 \\
17 & 12 & 15 & 6 & 21 & 27 \\
18 & 10 & 9 & 7 & 12 & 19 \\
19 & 7 & 5 & 4 & 8 & 12 \\
20 & 6 & 2 & 2 & 6 & 8 \\
21 & 0 & 2 & 0 & 2 & 2 \\
\hline Total & 65 & 64 & 34 & 95 & 129 \\
\hline
\end{tabular}

Tabla II. Cuestionamiento de autoimagen.

\begin{tabular}{ll}
\hline ¿Tengo algún diente chueco? & Si \\
¿Esto me molesta? & Siempre A veces Nunca \\
¿Cuando miro mis dientes, me gusta lo que veo? & Siempre A veces Nunca \\
El aspecto de mis dientes ¿Es un impedimento para relacionarme con mis pares? & Siempre A veces Nunca \\
Cuando sonrío ¿Me tapo la boca? & Siempre A veces Nunca \\
\hline
\end{tabular}




\section{RESULTADOS}

Los resultados muestran que el $32,6 \%$ de escolares presenta un grado de IED menor o igual a 25 , es decir, con ausencia de anomalías o maloclusión leve y en donde es innecesario o poco necesario un tratamiento. Un $26,3 \%$ es poseedor de maloclusión manifiesta (IED 26-30) y sería optativo un tratamiento; en un $19,4 \%$ es patente una maloclusión grave (IED 31-36) y es sumamente deseable el tratamiento; mientras que en un $21,7 \%$ la maloclusión es muy grave o discapacitante (IED $\geq 36$ ) y es obligatorio un tratamiento.

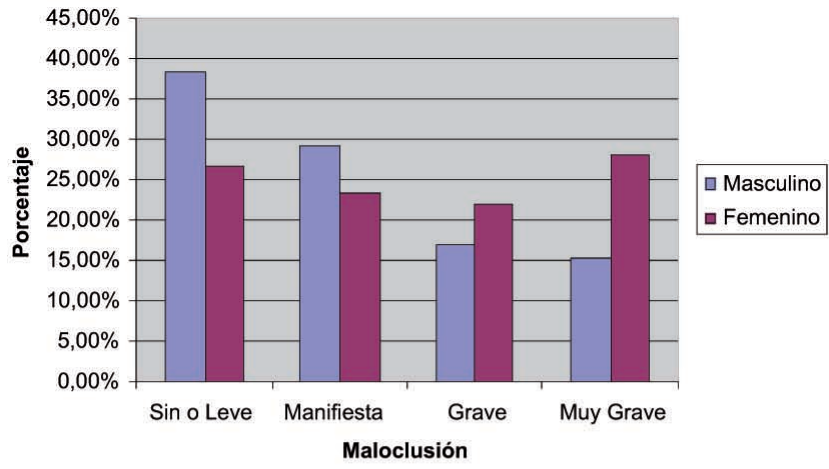

Fig. 1. Distribución porcentual del grado de maloclusion segun sexo en estudiantes del Liceo Interculutural de Ralco.

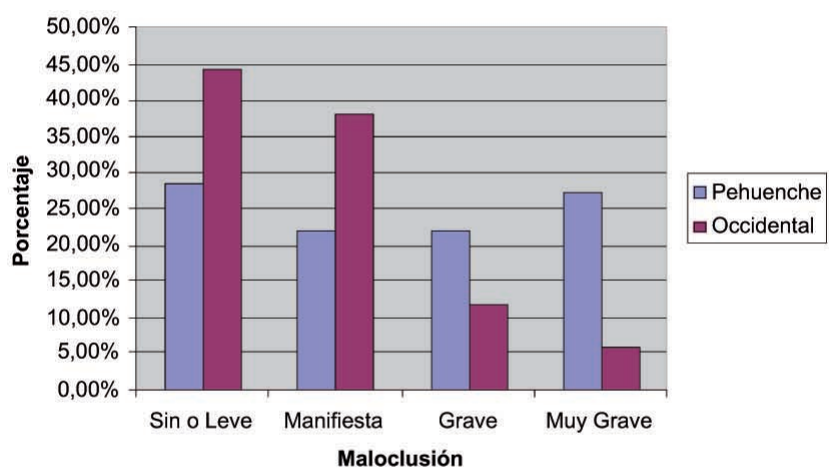

Fig. 3. Distribución porcentual del grado de maloclusion segun etnia en estudiantes del Liceo Interculutural de Ralco.

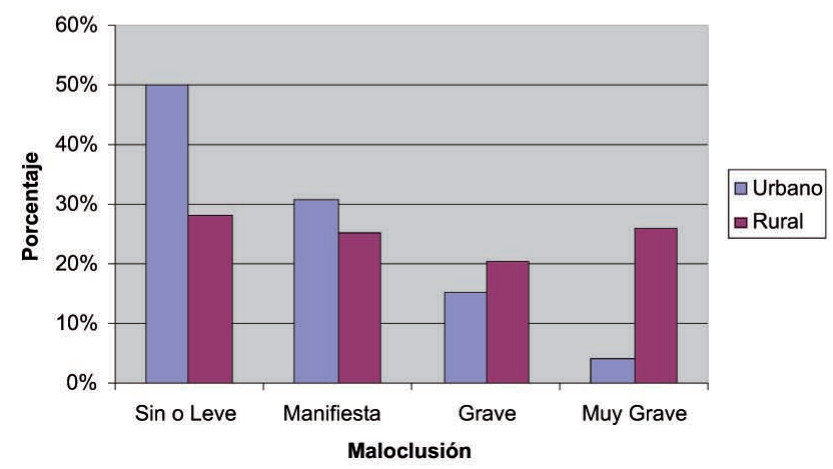

Fig. 4. Distribución porcentual del grado de maloclusion segun ruralidad en estudiantes del Liceo Interculutural de Ralco.
En la Fig. 1 se puede apreciar los distintos grados de maloclusiones según sexo, donde un $38,5 \%$ de los hombres no presentan maloclusiones o un nivel leve de estas mientras que en las mujeres este valor solo alcanza el $26,6 \%$; en tanto que en el nivel de maloclusiones muy graves o discapacitantes los hombres muestran un $15,4 \%$ en comparación a las mujeres con un $28,1 \%$; en general podemos apreciar una mayor prevalencia y gravedad de las maloclusiones en el grupo femenino, aunque no alcanza a considerarse estadísticamente significativa, $p=0,2008$.

La Fig. 2 muestra la distribucion segun etnia, acá encontramos un patrón bastante similar a la distribución por sexo, donde los estudiantes pehuenches presentan una mayor prevalencia y gravedad de maloclusiones, siendo estadísticamente significativa con un $p=0,0113$; aquí para el nivel de ausencia o maloclusión leve y el de muy grave los valores son de $28,4 \%$ y $27,4 \%$ para pehuenches y $44,1 \%$ y $5,9 \%$ para los jovenes occidentales, respectivamente.

En cuanto a la influencia de la ruralidad (Fig. 3), si bien en términos reales la comuna completa de Alto Biobio esta considerada como rural, para efectos prácticos se decidió considerar a la localidad de Ralco como urbana y al resto de la comunidades como rurales, aquí nuevamente encontramos un patrón muy similar a los anteriores donde el grupo más afectado es el rural; los valores encontrados para los jóvenes provenientes del sector rural fueron de $28,1 \%$ y $26,2 \%$ y para el sector urbano fue de $50 \%$ y $3,8 \%$ para los niveles de ausente o leve y muy graves en maloclusiones, respectivamente; la diferencia fue estadísticamente significativa con un $p=0,0395$.

En términos generales las alteraciones más frecuentes fueron el apiñamiento del sector anterior con un $86 \%, 31 \%$ con 1 arco dentario y $55 \%$ con ambos, de prevalencia y una relacion molar anormal con un $72 \%$.

En relación al cuestionario de autoimagen un $69,2 \%$ y un $81,2 \%$ de los hombres y mujeres respectivamente siente que tiene algun tipo maloclusion ("diente chueco"). Mientras que al $53,8 \%$ de los hombres esta situacion les molesta a veces o siempre, en el caso de las mujeres es en un $72 \%$. Al $53,2 \%$ de las mujeres y al $63,1 \%$ de los hombres esto les trae problemas para relacionarse con sus pares; el $30,7 \%$ de los hombres se tapa su boca cuando sonrie, este valor sube a $59,7 \%$ en las mujeres (Tabla III). 
Tabla II. Distribución porcentual de respuestas al Cuestionario de Autoimagen segun sexo en estudiantes del Liceo Intercultural de Ralco.

\begin{tabular}{lcccccccccc}
\hline & \multicolumn{2}{c}{ Pregunta 1 } & \multicolumn{2}{c}{ Pregunta 2 } & \multicolumn{2}{c}{ Pregunta 3 } & \multicolumn{2}{c}{ Pregunta 4 } & \multicolumn{2}{c}{ Pregunta 5 } \\
\hline Sexo & M & $\mathrm{F}$ & $\mathrm{M}$ & $\mathrm{F}$ & $\mathrm{M}$ & $\mathrm{F}$ & $\mathrm{M}$ & $\mathrm{F}$ & $\mathrm{M}$ & $\mathrm{F}$ \\
\hline Siempre & $69,2 \%$ & $81,2 \%$ & $24,6 \%$ & $23,2 \%$ & $18,5 \%$ & $15,6 \%$ & $10,8 \%$ & $9,4 \%$ & $1,5 \%$ & $4,7 \%$ \\
A veces & - & - & $29,2 \%$ & $50,4 \%$ & $64,6 \%$ & $48,4 \%$ & $52,3 \%$ & $43,8 \%$ & $29,2 \%$ & $54,7 \%$ \\
Nunca & $30,8 \%$ & $18,8 \%$ & $46,2 \%$ & $26,4 \%$ & $16,9 \%$ & $36,0 \%$ & $36,9 \%$ & $46,8 \%$ & $69,3 \%$ & $40,6 \%$ \\
\hline
\end{tabular}

\section{DISCUSIÓN}

El desarrollo de soluciones y estrategias en salud pública necesitará siempre comenzar con un diágnostico adecuado: prevalencias, factores de riesgo y protectores, reconocimiento de los grupos desfavorecidos, etc. Si bien en Chile se cuenta con un diágnostico actualizado en el que se incluye el componente de las maloclusiones (Soto et al.), se hace insuficiente para determinar que grupos están más proclives a desarrollar este tipo de patología y necesitan por tanto una priorización, en aras de la equidad.

En nuestro caso hemos encontrado una alta prevalencia, $67,4 \%$ de los jóvenes del Liceo de Ralco muestran a lo menos una maloclusión manifiesta y en el $21,7 \%$ esta es de carácter discapacitante, por lo que un tratamiento es obligatorio. Si comparamos estos valores con las estadísticas de países desarrollados la situacion es aún mas preocupante, un estudio con un grupo y metodología similar realizado en Granada, España (Baca-Garcia et al., 2004) encontró una prevalencia del $41,4 \%$ y un $9,9 \%$ para el nivel discapacitante; en cambio estudios en países latinoamericanos: Perú (Bernabé \& Flores-Mir, 2006), Brasil (Marques et al., 2005; Frãzao \& Narvai, 2006) y Cuba (Toledo et al., 2004) arrojan resultados más cercanos al nuestro, aunque siempre menores.

La correlación existente con el sexo, si bien no estadísiticamente significativa, muestra una tendencia hacia una mayor prevalencia y gravedad en las mujeres, al respecto solo encontramos un trabajo con resultados similares (Hamamci et al., 2009), pero en general la literatura no muestra diferencias claras en relación al género.

Las variables etnia y ruralidad se encuentran totalmente interrelaciones, ya que en la comuna la población que proviene de las comunidades (rural) es eminentemente pehuenche a diferencia de la del sector de Ralco que es mayoritariamente occidental, por esta razón no parece raro que ambas variables hayan sido estadísticamente signifitivas con un comportamiento bastante similar; en relación a esto no existe información en la literatura por lo que no podemos compararnos con otras realidades, aunque si existe una relación con los valores de morbilidad bucal encontrados en niños de 6 y 12 años de la misma comuna (Araya \& Valdés, 2008a; 2008b) donde los grupos rural y pehuenche presentaban valores de ceod y COPD mayores, especialmente en el componente de piezas temporales extraídas antes de su exfoliación normal lo que explicaria, en cierto grado, una mayor susceptibilidad al desarrollo de maloclusiones.

La segunda parte del trabajo consistió en la evaluación de la influencia de las maloclusiones sobre aspectos psicosociales de los jóvenes y nos centramos específicamente en determinar en que grado afectaban la relación con sus pares, al respecto la literatura ofrece una amplisima gama de opciones con instrumentos para medir esta situación (Bernabé et al., 2008; Hamamci et al.; Klages et al.; Taylor et al.; Agou et al.; Marques et al., 2006; Onyeaso \& Sanu; de Paula et al.), en nuestro caso preferimos desarrollar un breve cuestionario con preguntas directas y simples que nos aseguraran el entendimiento por parte de los estudiantes. Los resultados dan cuenta de una alta correlación entre la existencia y la percepción de la maloclusión en los jóvenes; tambien encontramos un efecto negativo en la confianza para relacionarse con sus compañeros, lo que se da mayormente en las mujeres coincidiendo con datos anteriores (Marques et al., 2006), aunque en el estudio se aprecia una inconsistencia ya que ante la consulta si los "dientes chuecos" molestan y si me tapo la boca para sonreir una mucho mayor proporción de mujeres respondió afirmativamente, pero ante la pregunta directa si esto era un impedimento para relacionarse con sus pares la proporción fue levemente mayor en hombres. 
En conclusión, la población estudiada muestra una prevalencia de maloclusiones del $67,4 \%$, con un $21,7 \%$ en el nivel muy grave o discapacitante, valores superiores a estadísticas nacionales e internacionales, siendo mayormente afectados las mujeres, la población rural y pehuenche.

La presencia de maloclusiones tiene un efecto negativo en la confianza de los jovenes para relacionarse con sus pares, especialmente en las mujeres.

Es necesario desarrollar estrategias tanto preventivas como curativas que logren dar solución a este problema, especialmente en los grupos más afectados.

\section{AGRADECIMIENTOS}

Quisieramos manifestar nuestro agradecimiento al Sr. Alvaro Basualto y la Srta. Karina Bahamondes, psicólogos; y a la Sra. Luisa Alegria y el Sr. Juan Canio, facilitadores interculturales del CESFAM Ralco por su ayuda en la confección del Cuestionario de Autoimagen.

CARTES-VELÁSQUEZ, R.; ARAYA, E. \& VALDÉS, C. Malocclusions and its psychosocial impact in intercultural highschool students. Int. J. Odontostomat., 4(1):65-70, 2010.

ABSTRACT: Malocclusions are regarded as the third oral disease with most prevalence and impact in the world, despite this there are few studies in Chile that address the issue and consider their impact on the quality of life and relationship to specific population groups. The aim of this study is to determine the prevalence and psychosocial impact on young people studying in a high school with indigenous people. We performed a descriptive cross-sectional study in 129 students, $73.6 \%$ pehuenche, of Ralco High-school; Dental Aesthetic Index was used to quantify the malocclusion magnitude and a Selfimage Questionnaire for assessing psychosocial aspect. The results show a prevalence of $67.4 \%$ and $21.7 \%$ for disabling malocclusions level, was significantly higher in pehuenche and rural population, this values are over national and international statistics. More than half of youngs have troubles to relating with their peers to cause of malocclusion. This requires the implementation of preventive and curative strategies providing a solution to this problem.

KEY WORDS: malocclusion, dental aesthetic, self-esteem, psychosocial, indigenous.

\section{REFERENCIAS BIBLIOGRÁFICAS}

Agou, S.; Locker, D.; Streiner, D. L. \& Tompson, B. Impact of self-esteem on the oral-health-related quality of life of children with malocclusion. Am. J. Orthod. Dentofacial Orthop., 134(4):484-9, 2008.

Araya, E. \& Valdés, C. Estado de salud bucal en escolares de 6 años de la comuna de Alto Biobio, 2008. Informe de Internado Asistencial. Universidad de Concepción, 2008a.

Araya, E. \& Valdés, C. Estado de salud bucal en escolares de 12 años de la comuna de Alto Biobio, 2008. Informe de Internado Asistencial. Universidad de Concepción, 2008b.

Baca-Garcia, A.; Bravo, M.; Baca, P.; Baca, A. \& Junco, P. Malocclusions and orthodontic treatment needs in a group of Spanish adolescents using the Dental Aesthetic Index. Int. Dent. J., 54(3):138-42, 2004.

Bernabé, E. \& Flores-Mir, C. Orthodontic treatment need in Peruvian young adults evaluated through dental aesthetic index. Angle Orthod., 76(3):41721, 2006.

Bernabé, E.; de Oliveira, C. M. \& Sheiham, A. Comparison of the discriminative ability of a generic and a condition-specific OHRQoL measure in adolescents with and without normative need for orthodontic treatment. Health Qual. Life Outcomes, 6:64, 2008.

de Paula, D. F.; Santos, N. C. M.; da Silva, E.; Nunes, M. F. \& Leles, C. R. Psychosocial Impact of Dental Esthetics on Quality of Life in Adolescents. Angle Orthod., 79(6):1188-93, 2009.

Frazão, P. \& Narvai, P. C. Socio-environmental factors associated with dental occlusion in adolescents. Am. J. Orthod. Dentofacial Orthop., 129(6):809-16, 2006.

Hägg, U.; McGrath, C. \& Zhang, M. Quality of Life and 
Orthodontic Treatment Need Related to Occlusal Indices. Dent. Bull., 12(10):8-12, 2007.

Hamamci, N.; Bas, aran, G. \& Uysal, E. Dental Aesthetic Index scores and perception of personal dental appearance among Turkish university students. Eur. J. Orthod., 31(2):168-73, 2009.

Klages, U.; Claus, N.; Wehrbein, H. \& Zentner, A. Development of a questionnaire for assessment of the psychosocial impact of dental aesthetics in young adults. Eur. J. Orthod., 28(2):103-11, 2006.

Marques, L. S.; Barbosa, C. C.; Ramos-Jorge, M. L.; Pordeus, I. A. \& Paiva, S. M. Prevalência da maloclusão e necessidade de tratamento ortodôntico em escolares de 10 a 14 anos de idade em Belo Horizonte, Minas Gerais, Brasil: enfoque psicossocial. Cad. Saúde Pública, 21(4):1099-106, 2005.

Marques, L. S.; Ramos-Jorge, M. L.; Paiva, S. M. \& Pordeus, I. A. Malocclusion: esthetic impact and quality of life among Brazilian schoolchildren. Am. J. Orthod. Dentofacial Orthop., 129(3):424-7, 2006.

OMS. Encuestas de salud bucodental. Métodos Básicos. $4^{a}$ Ed. Ginebra, Organización Mundial de la Salud, 1997.

Onyeaso, C. O. \& Sanu, O. O. Perception of personal dental appearance in Nigerian adolescents. Am. J. Orthod. Dentofacial Orthop., 127(6):700-6, 2005.

Soto, L.; Tapia, R.; Jara, G.; Rodriguez, G. \& Urbina, T. Diágnostico nacional de salud bucal del adolescente de 12 años y evaluación del grado de cumplimiento de los objetivos sanitarios de salud bucal 2000-2010. Santiago, Facultad de Odontología, Universidad Mayor, 2007.

Taylor, K. R.; Kiyak, A.; Huang, G. J.; Greenlee, G. M.; Jolley, C. J. \& King, G. J. Effects of malocclusion and its treatment on the quality of life of adolescents. Am. J. Orthod. Dentofacial Orthop., 136(3):382-92, 2009.

Toledo, L.; Machado, M.; Martinez, Y. \& Muñoz, M. Maloclusiones por el índice de estética dental (DAI) en la población menor de 19 años. Rev. Cubana Estomatol., 41(3), 2004.
Dirección para correspondencia:

Ricardo Cartes-Velásquez

CESFAM Ralco Alto Biobio

Villa Ralco s/n. Alto Biobio

CHILE

Email: cartesvelasquez@gmail.com

Recibido : 06-10-2009

Aceptado: 04-11-2009 